\title{
Nghiên cứu xây dựng phương pháp định lượng esomeprazol trong huyết tương thỏ bằng sắc ký lỏng khối phổ hai lẩn (LC-MS/MS)
}

\author{
Lê Minh Anh ${ }^{1}$, Nguyễn Thạch Tùng ${ }^{1 *}$, Nguyễn Như Nam ${ }^{1}$ \\ Bùi Quang Đông ${ }^{2}$, Phạm Thành Đạt ${ }^{1}$, Nguyễn Thị Hồng Ngọc ${ }^{2}$, Trần Cao Sơn ${ }^{2 *}$ \\ ${ }^{1}$ Truờng Đại học Dược Hà Nội, Việt Nam \\ ${ }^{2}$ Viện Kiểm nghiệm an toàn vệ sinh thực phẩm Quốc gia, Hà Nội, Việt Nam
}

(Ngày đến tòa soạn: 28/12/2020; Ngày chấp nhận đăng: 15/03/2021)

\section{Tóm tắt}

Phương pháp sắc ký lỏng khối phổ hai lần (LC-MS/MS) đã được phát triển để định lượng nông độ esomeprazol trong huyết tương thỏ. Kỹ thuật QuEChERS được sử dụng để chiết và làm sạch esomeprazol trong huyết tương. Sau đó, chất phân tích được tách bằng sắc ký lỏng pha đảo với cột $\mathrm{C} 18$ và định lượng bằng khối phổ ba tứ cực. Nguồn ion hóa ESI dương, chế độ theo dõi ion MRM đã được sử dụng trong nghiên cứu. Pantoprazol được sử dụng làm chất chuẩn nội cho esomeprazol. Giá trị sử dụng của phương pháp được xác nhận theo hướng dẫn của $\mathrm{ICH}$ về thẩm định phương pháp phân tích thuốc trong dịch sinh học. Kết quả thẩm định cho thấy phương pháp có độ đặc hiệu tốt, đường chuẩn được xây dựng trong khoảng nông độ $0,1-20 \mathrm{ng} / \mathrm{mL}$, giới hạn định lượng dưới là $0,1 \mathrm{ng} / \mathrm{mL}$; độ đúng và độ chính xác trong khoảng nông độ từ 0,1 đến $20 \mathrm{ng} / \mathrm{mL}$ đều đáp ứng theo yêu câuu của $\mathrm{ICH}$ và $\mathrm{AOAC}$. Hàm lượng chất phân tích trong mẫu huyết tương ổn định trong 3 chu kỳ đông - rã đông trong vòng 03 tháng và nông độ chất phân tích trong dịch chiết ổn định trong 24 giờ lưu giũ trong bộ tiêm mẫu. Phương pháp được áp dụng để định lượng nồng độ esomeprazol trong huyết tương thỏ và ứng dụng để so sánh sinh khả dụng của hai chế phẩm có chứa esomeprazol.

Tü khóa: Esomeprazol, pantoprazol, LC-MS/MS, huyết tương thỏ, QuEChERS.

\section{1. ĐĂT VẤN ĐỀ}

Esomeprazol (5-methoxy-2-\{[(4-methoxy-3,5-dimethylpyridin-2-yl) methyl $]$ sulfinyl $\}$ $1 \mathrm{H}$-benzimidazol) là hoạt chất thuộc nhóm ức chế bơm proton, được chỉ định điều trị loét dạ dày - tá tràng, trào ngược dạ dày - thực quản, hội chứng tăng tiết acid dịch vị, dự phòng loét dạ dày - tá tràng khi sử dụng thuốc chống viêm không steroid, do stress [1-2]. Do đặc tính kém bên trong môi trường acid dịch vị nên các chế phẩm chứa esomeprazol đều được bào chế dưới dạng thuốc giải phóng tại ruột [3].

Trên thế giới đã có nhiều nghiên cứu để xác định nông độ esomeprazol trong dịch sinh học bằng HPLC-UV hoặc LC-MS/MS. Với HPLC-UV, Talaat và cộng sự (2017) đã xây dựng phương pháp định lượng nông độ esomeprazol trong huyết tương và đạt được giới hạn định lượng dưới (LLOQ) rất thấp $0,092 \mathrm{ng} / \mathrm{mL}$ [4]. Tuy nhiên, phương pháp HPLC-UV thường có độ đặc hiệu thấp và phần lớn các nghiên cứu sử dụng LC-MS/MS để định lượng esomeprazol trong nền mẫu dịch sinh học. Chunduri và cộng sự (2016) đã nghiên cứu xây dựng phương

*Điện thoại: 0969748898

*Điện thoại: 0988683282
Email:nguyenthachtung@hup.edu.vn

Email:sontc@nifc.gov.vn 
pháp định lượng esomeprazol trong huyết tương bằng UPLC-MS/MS với LLOQ là $0,1 \mathrm{ng} / \mathrm{mL}$ [5]. Elkady và cộng sự (2018) cũng đã nghiên cứu định lượng nông độ esomeprazol trong huyết tương bằng LC-MS/MS với LLOQ là $20 \mathrm{ng} / \mathrm{mL}$, độ thu hồi từ 88,7 -104\% [6].

QuEChERS (quick, easy, cheap, effective, rugged, safe) là kỹ thuật xử lý mẫu để xác định dư lượng hóa chất bảo vệ thực vật trong mẫu thực phẩm do Anastassiades và cộng sự phát triển [7]. Cơ chế chung của kỹ thuật QuEChERS là làm tăng tính phân cực của pha nước khi thêm muối chiết từ đó tách pha dung môi hữu cơ khỏi pha nước, chất phân tích được chiết vào pha hữu cơ và tiến hành phân tích ở các bước tiếp theo. Gần đây, nhiều nghiên cứu đã ứng dụng kỹ thuật QuEChERS để phân tích hoạt chất trong dịch sinh học với nhiều ưu điểm vượt trội như độ nhạy tốt, tính đặc hiệu cao, thời gian phân tích nhanh, quá trình xử lý mẫu đơn giản.

Nghiên cứu này thực hiện xây dựng và thẩm định phương pháp định lượng esomeprazol trong huyết tương thỏ bằng LC-MS/MS kết hợp xử lý mẫu bằng kỹ thuật QuEChERS hạn chế ảnh hưởng của nển mẫu so với nhiều phương pháp định lượng trước đây, nghiên cứu này cũng đề cập đến độ ổn định của mẫu mà ít nghiên cứu đã công bố. Phương pháp được ứng dụng để phân tích esomeprazol trong huyết tương, phục vụ đánh giá sinh khả dụng của chế phẩm có chứa esomeprazol.

\section{VẬT LIỆU VÀ PHƯƠNG PHÁP NGHIÊN CỨU}

\section{1. Đối tượng và mẫu nghiên cứu}

Đối tượng nghiên cứu là esomeprazol trong huyết tương thỏ. Mẫu nghiên cứu gồm: mẫu trắng là mẫu huyết tương thỏ không có chất phân tích, mẫu chuẩn là mẫu chứa esomeprazol trong dung môi acetonitril, mẫu trắng thêm chuẩn là mẫu huyết tương có chứa chuẩn esomeprazol, mẫu thực là mẫu huyết tương có chứa esomeprazol được lấy và xử lý sau khi cho thỏ uống chế phẩm có chứa esomeprazol.

\subsection{Hóa chất, chất chuẩn và động vật nghiên cứu}

Chất chuẩn esomeprazol natri (SKS:0114302.01) hàm lượng 96,31\% được đóng gói trong lọ thủy tinh tối màu $(200 \mathrm{mg} / \mathrm{lọ})$, bảo quản ở điều kiện $2^{\circ} \mathrm{C}-8^{\circ} \mathrm{C}$, tránh ánh sáng; và chuẩn nội pantoprazol (SKS: 0114306.01) có nguôn gốc từ Viện Kiểm nghiệm thuốc Trung Ương, muối magnesi sulfat khan có nguôn gốc từ Đức (TCNSX), muối natri clorid có nguồn gốc từ Trung Quốc (TCNSX), các dung môi gồm acetonitril $(\mathrm{ACN})$, methanol $(\mathrm{MeOH})$ và acid formic có nguôn gốc từ Merck (Đức) đều đạt tiêu chuẩn tinh khiết dùng cho HPLC và LC-MS.

Động vật nghiên cứu là thỏ trắng giống đực trưởng thành (4 - 6 tháng tuổi), khối lượng khoảng $2 \mathrm{~kg}$, được nhập về trước khi tiến hành thí nghiệm 02 tuần và nuôi trong Phòng thí nghiệm dược lý Trường Đại học Dược Hà Nội.

\subsection{Thiết bị và dụng cụ}

Hệ thống sắc ký lỏng khối phổ hai lần gồm thiết bị khối phổ SCIEX ExionLC ${ }^{\mathrm{TM}} \mathrm{AD}$ Triplequad $6500^{+}$(Mỹ) kết nối với sắc ký lỏng LC 20AD XR của SCIEX (Mỹ). Một số thiết bị phụ trợ khác bao gồm máy ly tâm Mikro 220 (Mỹ); máy ly tâm Hermle Z200A (Mỹ); cân phân tích (có độ chính xác 0,1mg) XS105 (Metter Toledo) và các thiết bị, dụng cụ khác trong phòng thí nghiệm. 


\subsection{Phương pháp nghiên cứu}

\subsubsection{Khảo sát điều kiện sắc kýlỏng khối phổ LC-MS/MS để phân tích esomeprazol}

Để tối ưu hóa điều kiện phân tích, tiêm trực tiếp dung dịch chuẩn esomeprazol 1 $\mu \mathrm{g} / \mathrm{mL}$ và pantoprazol $1 \mu \mathrm{g} / \mathrm{mL}$ vào detector $\mathrm{MS} / \mathrm{MS}$ với nguôn ion hóa phun điện tử $\mathrm{ESI}$ dương. Chọn chế độ khảo sát tự động để bắn phá ion phân tử thành các ion con, lựa chọn ion con có cường độ tín hiệu cao nhất để định lượng và ion con có cường độ thấp hơn để định tính. Tối ưu hóa năng lượng bắn phá $(\mathrm{CE})$ tự động theo phần mềm Analyst 1.7 của thiết bị. Đối với điều kiện LC sử dụng cột sắc ký Agilent Eclipse Plus C18 (150mm x 2,1mm), kích thước hạt nhôi 3,5 $\mu \mathrm{m}$, tốc độ dòng $0,5 \mathrm{~mL} /$ phút, thể tích tiêm $10 \mu \mathrm{L}$, thành phần pha động gồm $\mathrm{ACN}$ và dung dịch acid formic $0,1 \%$, tốc độ dòng là $0,5 \mathrm{~mL} /$ phút và tiến hành khảo sát điều kiện chương trình gradient rửa giải chất phân tích.

\subsubsection{Khảo sát điều kiện xủ lý mẫu}

Điều kiện xử lý mẫu được tối ưu thông qua việc khảo sát một số quy trình sau:

Quy trình 1: Sử dụng kỹ thuật chiết lỏng - lỏng [5]. Hút chính xác $475 \mu \mathrm{L}$ huyết tương cho vào ống ly tâm $2 \mathrm{~mL}$. Thêm chính xác $25 \mu \mathrm{L}$ dung dịch chuẩn nội $100 \mathrm{ng} / \mathrm{mL}$ và $500 \mu \mathrm{L}$ dung môi chiết. Lắc xoáy trong 1 phút. Ly tâm với tốc độ 13.000 vòng/phút trong 5 phút. Hút $250 \mu \mathrm{L}$ lớp dung môi phía trên, thổi khô bằng khí $\mathrm{N}_{2}$, hòa tan lại cắn bằng $250 \mu \mathrm{L}$ acetonitril. Khảo sát một số môi trường chiết với quy trình 1: ethyl acetat (EtOAc), diethyl ether (DE), hỗn hợp methyl tert buthyl ether (MTBE) : ethyl acetat $(8: 2)$, hỗn hợp methyl tert buthyl ether: ethyl acetat $(5: 5)$.

Quy trình 2: Sử dụng kỹ thuật chiết QuEChERS [7]. Hút chính xác $475 \mu \mathrm{L}$ huyết tương cho vào ống ly tâm $2 \mathrm{~mL}$. Thêm chính xác $25 \mu \mathrm{L}$ dung dịch chuẩn nội pantoprazol $100 \mathrm{ng} / \mathrm{mL}$ và $500 \mu \mathrm{L}$ acetonitril. Lắc xoáy trong vòng 1 phút. Thêm muối chiết vào mỗi ống ly tâm. Tiếp tục lắc xoáy trong 1 phút. Sau đó ly tâm với tốc độ 13.000 vòng/phút trong 10 phút. Hút $200 \mu \mathrm{L}$ lớp dịch lỏng trên cùng vào lọ mẫu, tiến hành định lượng nông độ dược chất bằng phương pháp sắc ký lỏng khối phổ LC-MS/MS. Khảo sát các loại muối chiết với quy trình 2: $\mathrm{MgSO}_{4}, \mathrm{MgSO}_{4}$ $+\mathrm{NaCl}, \mathrm{MgSO}_{4}+\mathrm{PSA}, \mathrm{CH}_{3} \mathrm{COONH}_{4}, \mathrm{MgSO}_{4}+\mathrm{C}_{18}$ và $\mathrm{NH}_{4} \mathrm{Cl}$.

\subsubsection{Thâm định phương pháp}

Tiến hành thẩm định phương pháp đã tối ưu thông qua các thông số cơ bản gồm: độ đặc hiệu; giới hạn định lượng dưới (LLOQ); đường chuẩn; độ đúng và độ chính xác; độ thu hồi; độ ổn định của huyết tương trong thời gian dài, sau 3 chu kì đông - rã đông và sau xử lý - bảo quản trong bộ phận tiêm mẫu tự động. Các kết quả được đánh giá so sánh với các quy định theo Dược điển Việt Nam V, ICH, FDA và $\mathrm{AOAC}$ [8-11].

\subsubsection{Nghiên cứu dược động học}

Hai chế phẩm gồm chế phẩm đối chiếu bột pha hỗn dịch Nexium 10 mg hàm lượng 99,7\% so với nhãn (LOT RCWB/EXP 31-03-20) và chế phẩm bào chế micropellet chứa esomeprazol dạng bào chế giải phóng tại ruột hàm lượng 12,4\% được cho uống trên thỏ để xây dựng mô hình dược động học không ngăn. Thỏ được chia làm 02 nhóm, mỗi nhóm 03 con, không cho ăn vào ngày trước ngày tiến hành nghiên cứu, mức liều tham khảo hướng dẫn của FDA [12]. Nhóm 1 cho uống chế phẩm đối chiếu với liều $2,5 \mathrm{mg} / \mathrm{kg}$. Nhóm 2 cho uống micropellet với liêu $2,5 \mathrm{mg} / \mathrm{kg}$. Sau khi cho thỏ uống thuốc, tiến hành lấy $3 \mathrm{~mL}$ máu ở tĩnh mạch tai thỏ cho vào ống có chứa EDTA tại các thời điểm xác định 0 giờ; 0,25 giờ; 0,5 giờ; 1 giờ; 1,5 giờ; 2 giờ; 
2,5 giờ; 3 giờ; 3,5 giờ; 4 giờ; 6 giờ; 8 giờ; 24 giờ. Ly tâm mẫu máu với tốc độ 6.000 vòng/phút trong 15 phút. Thu huyết tương, bảo quản ở $-10^{\circ} \mathrm{C}$ trước khi phân tích.

\subsubsection{Phương pháp xủ lý số liẹu}

Xử lý các số liệu thống kê bằng phần mềm Microsoft Excel 2010. Nông độ esomeprazol trong huyết tương được tính toán bằng phần mềm Analyst 1.7 trên thiết bị sắc kýlỏng khối phổ.

\section{KẾT QUẢ VÀ BÀN LUẬN}

\subsection{Tối ưu hóa phương pháp phân tích esomeprazol bằng LC-MS/MS}

\subsubsection{Khảo sát điều kiện về khối phổ}

Thiết bị khối phổ ba tứ cực với nguôn ion hóa phun điện tử, chế độ ion dương được sử dụng để khảo sát điều kiện ion mẹ và ion con tối ưu. Kết quả được trình bày trong Bảng 1 .

Bảng 1. Các ion phân tử và ion con trong phân tích khối phổ

\begin{tabular}{ccccc}
\hline Chất phân tích & $\begin{array}{c}\text { Mảnh me } \\
(\boldsymbol{m} / \boldsymbol{z})\end{array}$ & $\begin{array}{c}\text { Mảnh con } \\
(\boldsymbol{m} / \boldsymbol{z})\end{array}$ & CE $(\boldsymbol{e V})$ & Ghi chú \\
\hline & & 198,0 & 25 & Định tính $\left(26,6 \%^{*}\right)$ \\
Esomeprazol & 346,1 & 168,0 & 21 & Định lượng \\
& & 151,0 & 29 & Định tính $\left(44,8 \% \%^{*}\right)$ \\
& & 138,0 & 43 & Định tính $\left(82,9 \%^{*}\right)$ \\
Pantoprazol & 384,0 & 200,0 & 19 & Định lượng \\
(chuẩn nội) & & 153,0 & 23 & Định tính $\left(4,7 \%^{*}\right)$ \\
\hline
\end{tabular}

* Tỷ lệ ion định tính so với ion định lượng

Các thông số phân tích khối phổ khác được tối ưu bao gôm: Điện thế đầu phun 5.500V; nhiệt độ đầu phun $450^{\circ} \mathrm{C}$; áp suất khí bổ trợ 30 psi; áp suất khí mang 8 psi.

\subsubsection{Khảo sát điều kiện sắc ký}

Sau khi lựa chọn được ion phân tử, ion con và các điều kiện tối ưu, tiến hành phân tích dung dịch chuẩn esomeprazol có nồng độ $50 \mathrm{ng} / \mathrm{mL}$ và nội chuẩn pantoprazole có nông độ $50 \mathrm{ng} / \mathrm{mL}$.

Gradient pha động gồm 2 kênh acetonitril và acid formic $0,1 \%$ được khảo sát để tối ưu, tốc độ dòng 0,5 mL/phút, thể tích tiêm $10 \mu \mathrm{L}$, cột sắc ký Agilent Eclipse Plus C18 (150 mm x 2,1 mm), kích thước hạt nhôi 3,5 m. Kết quả điều kiện gradient tối ưu được thể hiện trong Bảng 2.

Bảng 2. Chương trinh dung môi của phương pháp sắc ký lỏng khối phổ

\begin{tabular}{ccc}
\hline \multirow{2}{*}{ Thò̀i gian (phút) } & \multicolumn{2}{c}{ Pha động } \\
\cline { 2 - 3 } & Acid formic $\mathbf{0 , 1} \% \mathbf{H}_{2} \mathbf{O}(\%)$ & ACN (\%) \\
\hline 0,00 & 10 & 90 \\
2,50 & 10 & 90 \\
5,00 & 90 & 10 \\
7,00 & 90 & 10 \\
7,50 & 10 & 90 \\
10,00 & 10 & 90 \\
\hline
\end{tabular}


Hình 1 thể hiện sắc ký đô của esomeprazol và pantoprazol trong dung môi $\mathrm{ACN}$ sau khi đã xác định được các điều kiện LC-MS/MS. Pic thu được cân đối, nhọn, không có hiện tượng dính pic, điều kiện phân tích là phù hợp để tiến hành định lượng esomeprazol trong huyết tương.

blk-spk5.3 - Esomeprazole2 (QC) 346.100/188.000 Da - sample 28 of 40 from Dat Area: $4.79 \mathrm{e}+005$ counts Height: $1.20 \mathrm{e}+005$ cos RT: $4.39 \mathrm{~min}$

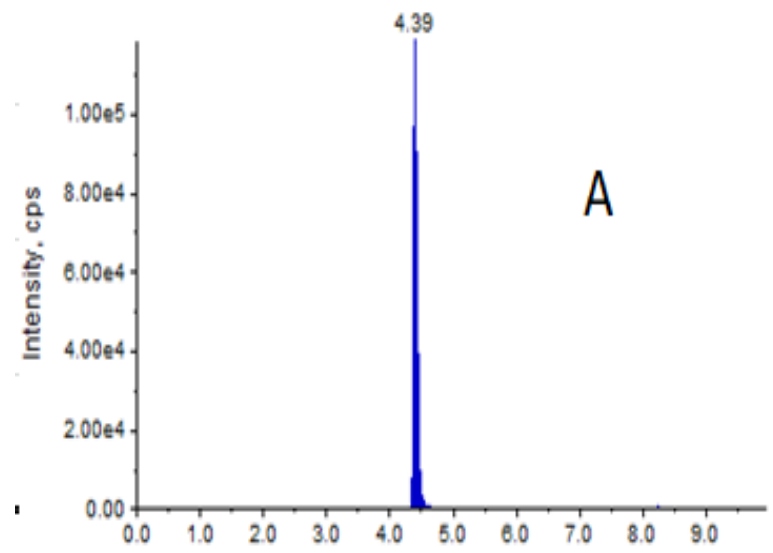

blk-spil5.3 - Pantoprazole2(IS) (QC) 384.000/200.000 Da - sample 26 of 40 from Avea: $3.39 \mathrm{e}+006$ counts Height: $7.46 \mathrm{e}+005$ cps RT: $4.55 \mathrm{~min}$

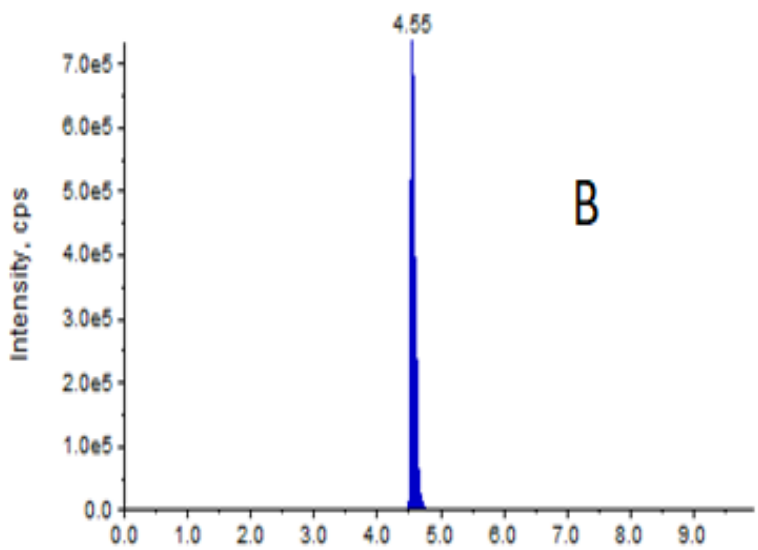

Hinh 1. Sắc ký đồ của chất chuân esomeprazol $5 \mathrm{ng} / \mathrm{mL}$ (A) và chuân nội pantoprazole $5 \mathrm{ng} / \mathrm{mL}$ (B) pha trong dung môi acetonitril

\subsection{Khảo sát quy trình xử lý mẫu}

\subsubsection{Lư chọn quy trình}

Quy trình 1 sử dụng một số loại dung môi để khảo sát được tham khảo [5]: ethyl acetat (EtOAc) QT 1.1, diethyl ether (DE) QT 1.2, hỗn hợp methyl tert buthyl ether (MTBE): ethyl acetat $(8: 2)$ QT 1.3, hỗn hợp methyl tert buthyl ether : ethyl acetat (5:5) QT 1.4. Quy trình 2 là phương pháp QuEChERS, sử dụng muối chiết là $\mathrm{MgSO}_{4} \mathrm{QT} 2$. Với quy trình chuẩn bị mẫu như đã trình bày ở mục 2.4.2. Các kết quả được trình bày ở Hình 2 và Bảng 3 .

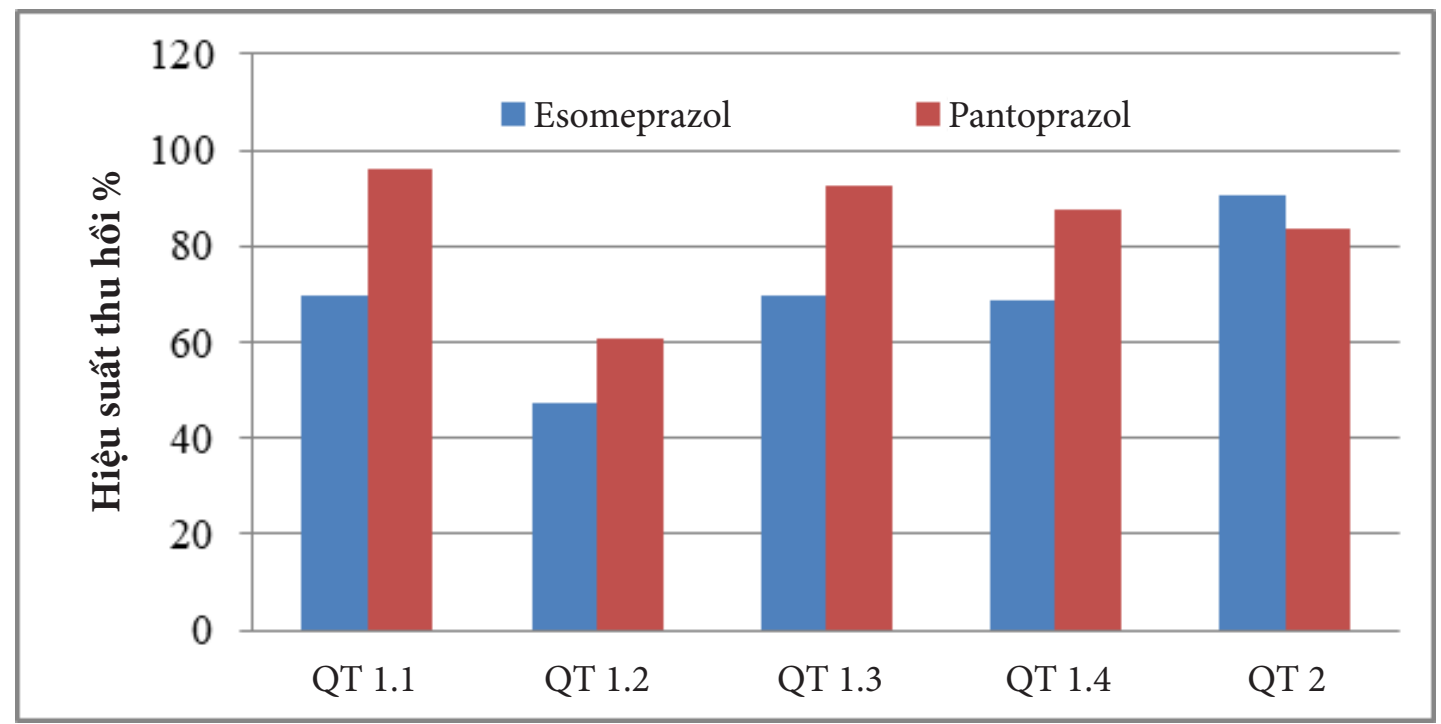

Hình 2. Kết quả khảo sát quy trình chiết 
Bảng 3. Hiệu suất thu hồi và ảnh hưởng của nền mẫu với các loại dung môi trong phuơng pháp chiết lỏng - lỏng

\begin{tabular}{cccc}
\hline \multirow{2}{*}{ Loại dung môi } & \multicolumn{2}{c}{ Hiệu suất thu hồi (\%) } & Ảnh huiởng của nền \\
\cline { 2 - 3 } & Chuần & Nội chuân & mấu (\% N) \\
\hline EtOAc & 69,91 & 96,20 & 24,68 \\
DE & 47,29 & 60,53 & 52,71 \\
MTBE:EtOAc (8 : 2) & 69,53 & 92,83 & 16,65 \\
MTBE:EtOAc (5 : 5) & 68,66 & 87,51 & 19,25 \\
\hline
\end{tabular}

Chú thích: Ảnh hưởng của nền mẫu: $N=\left(S_{c}-S_{s p k}\right) / S_{c} \times 100 \%$

Trong đó: $S_{c}$ là diện tích pic chất phân tích pha trong dung môi.

$\mathrm{S}_{\text {spk }}$ là diện tích pic chất phân tích được thêm vào trước quy trình chiết.

Hiệu suất thu hồi với tất cả các loại dung môi ở cả chất chuẩn $(47,3$ - 69,9\%), chất chuẩn nội $(60,5-96,2 \%)$ và giữa chất chuẩn so với chất chuẩn nội trong quy trình 1 đều thấp, ảnh hưởng của nền mẫu trong quy trình 1 lên kết quả lớn $(16,7-52,7 \%)$. Ngược lại, quy trình 2 cho hiệu suất thu hôi ở cả chất chuẩn $(90,7 \%)$ và chất chuẩn nội $(83,8 \%)$ đều cao, đặc biệt là hiệu suất thu hồi chất chuẩn tăng đáng kể so với quy trình 1 . Việc không sử dụng bước làm sạch cho hiệu suất cao trong thu hôi pantoprazol với dung môi ethyl acetat $(96,2 \%)$ hay MTBE: EtOAc (8 :2) $(92,8 \%)$ nhưng ảnh hưởng của nền mẫu cũng cao do dịch chiết vẫn còn nhiêu tạp. Kỹ thuật QuEChERS cho hiệu suất thu hồi cao, hạn chế ảnh hưởng của nền mẫu, do đó quy trình xử lý mẫu 2 (kỹ thuật QuEChERS) được lựa chọn để tiến hành các nghiên cứu tiếp theo.

\subsubsection{Khảo sát muối chiết}

Loại muối chiết và lượng muối chiết có ảnh hưởng lớn đến độ thu hồi. Cố định nông độ thêm chuẩn là $50 \mathrm{ng} / \mathrm{mL}$ để thực hiện các bước khảo sát. Một số muối chiết thông thường sử dụng cho phương pháp QuEChERS được tiến hành khảo sát: $\mathrm{MgSO}_{4}, \mathrm{MgSO}_{4}+\mathrm{NaCl}, \mathrm{MgSO}_{4}+$ $\mathrm{PSA}, \mathrm{MgSO}_{4}+\mathrm{C}_{18}, \mathrm{CH}_{3} \mathrm{COONH}_{4}, \mathrm{NH}_{4} \mathrm{Cl}$ [7].

Sau khi hút $450 \mu \mathrm{L}$ huyết tương trắng đã được xác định là không có esomeprazol, thêm $25 \mu \mathrm{L}$ dung dịch chuẩn nội $1 \mu \mathrm{g} / \mathrm{mL}$ và $25 \mu \mathrm{L}$ dung dịch chất chuẩn $1 \mu \mathrm{g} / \mathrm{mL}$, thêm $500 \mu \mathrm{L}$ acetonitril và tiến hành chiết mẫu với 06 loại hỗn hợp muối chiết khác nhau: $0,2 \mathrm{~g} \mathrm{MgSO}_{4}(\mathrm{QT}$ 2.1); 0,2 $\mathrm{g} \mathrm{MgSO}_{4}+$ 0,05 g NaCl (QT 2.2); 0,2 $\mathrm{g} \mathrm{MgSO}_{4}+$ 0,05 g PSA (QT 2.3); 0,2 g MgSO + 0,05 $\mathrm{g} \mathrm{C}_{18}$ (QT 2.4); 0,2 $\mathrm{g} \mathrm{CH}_{3} \mathrm{COONH}_{4}$ (QT 2.5); 0,2 $\mathrm{g} \mathrm{NH}_{4} \mathrm{Cl}$ (QT 2.6), tiến hành các bước như quy trình. Kết quả thu được thể hiện trong Hình 3.

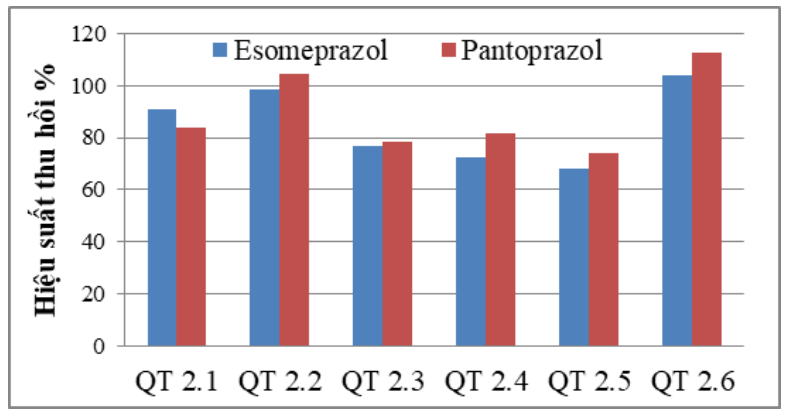

Hình 3. Hiệu suất thu hồi của esomeprazol theo các quy trình xủ lý mẫu khác nhau 
Sử dụng muối chiết $\mathrm{NH}_{4} \mathrm{Cl}, \mathrm{CH}_{3} \mathrm{COONH}_{4}$ làm cho thể tích lớp acetonitril tách ra ít, dẫn tới không chiết được hoàn toàn chất phân tích ra khỏi pha nước. Với các muối chiết còn lại $\mathrm{MgSO}_{4}, \mathrm{MgSO}_{4}+\mathrm{NaCl}, \mathrm{MgSO}_{4}+\mathrm{PSA}_{2} \mathrm{MgSO}_{4}+\mathrm{C}_{18}$ cho thể tích lớp acetonitril tương đương nhau, với cách sử dụng hỗn hợp muối $\mathrm{MgSO}_{4}+\mathrm{NaCl}$ cho hiệu suất thu hồi mẫu chuẩn cao nhất. Do đó, hỗn hợp muối $\mathrm{MgSO}_{4}+\mathrm{NaCl}$ được sử dụng cho các thí nghiệm tiếp theo.

Như vậy, quy trình xử lý mẫu huyết tương được tối ưu như sau: Hút chính xác $475 \mu \mathrm{L}$ huyết tương cho vào ống ly tâm $2 \mathrm{~mL}$. Thêm chính xác $25 \mu \mathrm{L}$ dung dịch chuẩn nội pantoprazol $100 \mathrm{ng} / \mathrm{mL}$ và $500 \mu \mathrm{L}$ acetonitril. Thực hiện lắc xoáy trong vòng 1 phút. Thêm vào mỗi ống ly tâm hỗn hợp muối chiết $0,2 \mathrm{~g} \mathrm{MgSO}_{4}+0,05 \mathrm{~g} \mathrm{NaCl}$. Tiếp tục lắc xoáy trong 1 phút. Sau đó ly tâm với tốc độ 13.000 vòng/phút trong 10 phút. Hút $200 \mu \mathrm{L}$ lớp dịch lỏng trên cùng vào lọ mẫu, tiến hành định lượng nồng độ esomeprazol bằng phương pháp sắc ký lỏng khối phổ LC-MS/MS.

\subsection{Thẩm định phương pháp phân tích}

\subsection{1. Độ đặc hiệu}

Đánh giá độ đặc hiệu của phương pháp qua các tiêu chí sau:

+ Tính số điểm IP (IP - Identification point): Theo cách tính điểm IP đối với mỗi ion mẹ là 1 điểm, mỗi ion con là 1,5 điểm. Kết quả thu được trên mẫu chuẩn và trên mẫu trắng thêm chuẩn cho thấy esomeprazol và pantoprazol đều có số điểm $\mathrm{IP}=5,5 \geq 4$; thỏa mãn yêu cầu phân tích khối phổ LC-MS/MS của AOAC [10].
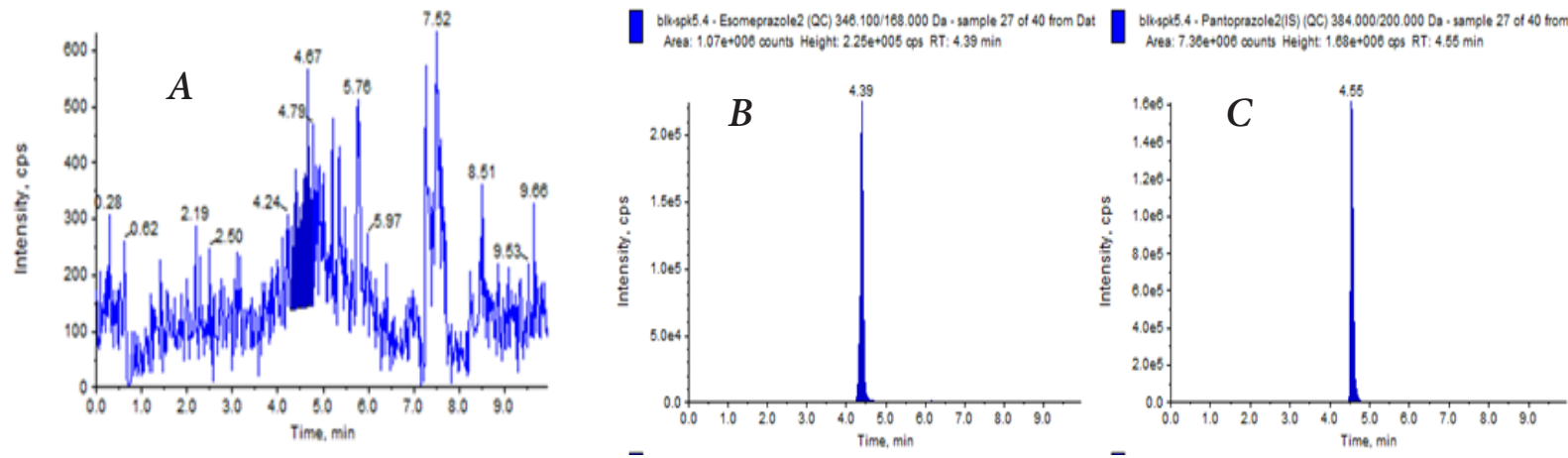

Hinh 4. Sắc ký đồ thẩm định tính đặc hiệu của phương pháp phân tích xác định nông độ esomeprazol trong huyết tương bằng thiết bị LC-MS/MS (A: Sắc ký đồ của mẫu trắng,

$B$ : Sắc ký đồ của mẫu trắng thêm esomeprazol chuân,

C: Sắc ký đồ của mẫu trắng thêm chuẩn nội pantoprazol chuân)

+ Phân tích mẫu trắng $(\mathrm{n}=6)$, mẫu trắng thêm chuẩn esomeprazol $5 \mathrm{ng} / \mathrm{mL}$ và pantoprazol $5 \mathrm{ng} / \mathrm{mL}$ theo phương pháp đã được xây dựng. Trên sắc ký đồ của mẫu huyết tương trắng (Hình $4 \mathrm{~A}$ ), tại thời điểm 4,39 phút và 4,55 phút trùng với thời gian lưu của esomeprazol và pantoprazol (Hình $4 \mathrm{~B}$ và $4 \mathrm{C}$ ) không xuất hiện các pic có mảnh phổ khối $\mathrm{m} / \mathrm{z}=346,1 / 168$ (đặc trưng của esomeprazol), mảnh phổ khối $\mathrm{m} / \mathrm{z}=384 / 200$ (đặc trưng cho pantoprazol). Do vậy, phương pháp phân tích có độ đặc hiệu với esomeprazol và pantoprazol theo các quy định của phương pháp phân tích thuốc trong dịch sinh học $[9,11]$.

\subsection{2. Đường chuân và khoảng tuyến tính}

Tiến hành thêm chuẩn vào huyết tương trắng ở các mức nồng độ từ 0,1 đến $20,0 \mathrm{ng} / \mathrm{mL}$ có thêm $5 \mathrm{ng} / \mathrm{mL}$ chuẩn nội pantoprazol và phân tích trên LC-MS/MS. Xây dựng đường phụ 
thuộc giữa nồng độ esomeprazol thu được với tỷ lệ giữa diện tích pic chất chuẩn và diện tích pic chuẩn nội. Đường chuẩn được lập theo phần mêm của thiết bị, theo phương pháp hồi quy tuyến tính, sử dụng hệ số tỷ trọng $1 / \mathrm{x}^{2}$. Các kết quả cho thấy trong khoảng nồng độ đã khảo sát có sự tương quan tuyến tính nông độ với tỷ lệ tín hiệu của esomeprazol và chuẩn nội, phương trình hồi quy tuyến tính thu được là $\mathrm{y}=0,0345 \mathrm{x}+0,0016$, với hệ số tương quan tuyến tính $\mathrm{r}^{2}>0,99$ và độ chệch $<15 \%$ ở tất cả các giá trị nồng độ đạt yêu cầu cho phép theo quy định của phương pháp pháp phân tích thuốc trong dịch sinh học $[9,11]$.

\subsection{3. Đánh giá ảnh hưởng nền}

Ảnh hưởng nền của phương pháp được đánh giá thông qua so sánh đường chuẩn pha trên nền mẫu và trên nền dung môi, trong khoảng nông độ 0,1 đến $20,0 \mathrm{ng} / \mathrm{mL}$. Kết quả thu được dựa trên phương pháp hồi quy tuyến tính và được lập trên phần mềm của thiết bị phân tích. Phương trình hồi quy của mẫu chuẩn là $\mathrm{y}=0,0287 \mathrm{x}+0,0013, \mathrm{r}^{2}>0,99$; phương trình hồi quy của mẫu trắng thêm chuẩn là $y=0,0345 \mathrm{x}+0,0016, \mathrm{r}^{2}>0,99$. Hệ số góc của 2 đường chuẩn thu được lệch nhau $16,8 \%$ đạt yêu cầu cho phép theo quy định của phương pháp pháp phân tích thuốc trong dịch sinh học (dưới 20\%) [8].

\subsubsection{Giới hạn định lượng diới (LLOQ)}

Phân tích mẫu huyết tương trắng và mẫu huyết tương chứa esomeprazol với nông độ 0,1 $\mathrm{ng} / \mathrm{mL}$. Xác định diện tích pic esomeprazol và chuẩn nội pantoprazol của mẫu LLOQ và xác định nồng độ esomeprazol có trong các mẫu từ đường chuẩn. Kết quả thẩm định cho thấy tỷ lệ đáp ứng của pic esomeprazol trong mẫu LLOQ $(0,1 \mathrm{ng} / \mathrm{mL})$ so với đáp ứng tại thời gian lưu trong mẫu huyết tương trắng là 9,0 (lớn hơn 5).

\subsection{5. Độ đúng và độ chính xác}

Độ đúng và độ chính xác được xác định đông thời bằng cách sử dụng tối thiểu 3 nông độ của mẫu chuẩn kiểm tra, 1 nông độ gần với giá trị nhỏ nhất của đường chuẩn, 1 nồng độ gần với giới hạn trên của đường chuẩn và 1 nông độ gần điểm giữa. Tiến hành thực hiện đánh giá bằng cách thêm chuẩn vào mẫu huyết tương trắng ở 3 mức nông độ khác nhau là $0,1 \mathrm{ng} / \mathrm{mL}$; $5 \mathrm{ng} / \mathrm{mL}$ và $20 \mathrm{ng} / \mathrm{mL}(\mathrm{n}=6)$, nồng độ chuẩn nội pantoprazol là $5 \mathrm{ng} / \mathrm{mL}$, phân tích theo quy trình đã xây dựng. Các kết quả phân tích được thể hiện ở Bảng 4.

Bảng 4. Độ đúng và độ chính xác của phương pháp LC-MS/MS

\begin{tabular}{|c|c|c|c|}
\hline Nồng độ & $0,1 \mathrm{ng} / \mathrm{mL}$ & $5 n g / m L$ & $20 \mathrm{ng} / \mathrm{mL}$ \\
\hline Độ đúng (\%) & 61,3 & 99,2 & 91,9 \\
\hline Độ chính xác (\%) & 4,69 & 2,41 & 3,22 \\
\hline \multicolumn{4}{|c|}{$\begin{array}{l}\text { Các kết quả này cho thấy phương pháp có độ chính xác đáp ứng yêu câu theo quy định } \\
\text { phương pháp phân tích thuốc trong dịch sinh học (RSD\% không vượt quá } 15 \%)[9,11] \text {. Độ } \\
\text { g thể hiện qua độ thu hôi tại } 0,1 \mathrm{ng} / \mathrm{mL} \text { vượt quá giới hạn theo quy định của } \mathrm{ICH}(80-120 \%) \text {, } \\
\text { ng đáp ứng yêu câuu theo quy định của } \mathrm{AOAC} \text { (độ thu hồi } 60-115 \% \text { ) [10]. Hàm lượng khảo } \\
\text { hấp }(0,1 \mathrm{ng} / \mathrm{mL}) \text { có thể gây ảnh hưởng đến kết quả xác định độ thu hồi; đây là một điểm hạn } \\
\text { của phương pháp và cân được khắc phục trong các nghiên cứu tiếp theo. }\end{array}$} \\
\hline
\end{tabular}




\subsection{6. Độ ôn địh}

Khảo sát 02 lô mẫu tại LQC (mẫu kiểm tra nồng độ thấp $0,1 \mathrm{ng} / \mathrm{mL}$ ) và $\mathrm{HQC}$ (mẫu kiểm tra nồng độ cao $20 \mathrm{ng} / \mathrm{mL}$ ) cho các phép thẩm định trong thời gian dài, sau 3 chu kì đông - rã đông, sau xử lí - bảo quản trong lọ mẫu đặt trong bộ tiêm mẫu tự động 24 giờ. Tiến hành phân tích theo quy trình đã xây dựng, kết quả độ ổn định của mẫu huyết tương $(n=3)$ được thể hiện trong Hình 5 và Hình 6.

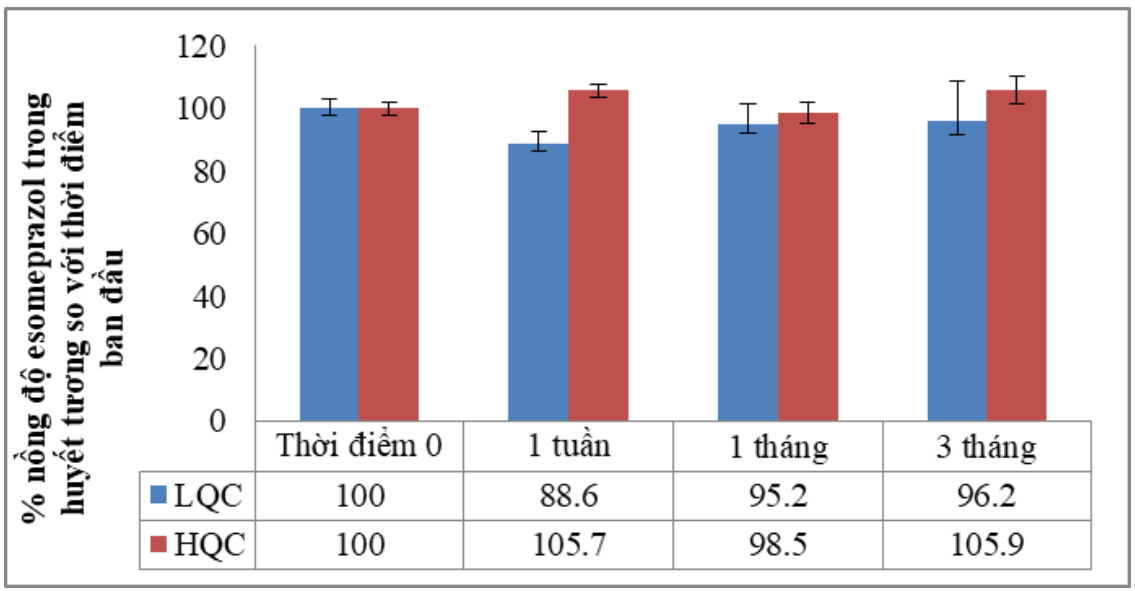

Hinh 5. Kết quả độ ổn định dài hạn và 3 chu kì đông - rã đông của mẫu (rã lân 1, lân 2 và lần 3 tương ứng với thời gian 1 tuân, 1 tháng và 3 tháng)

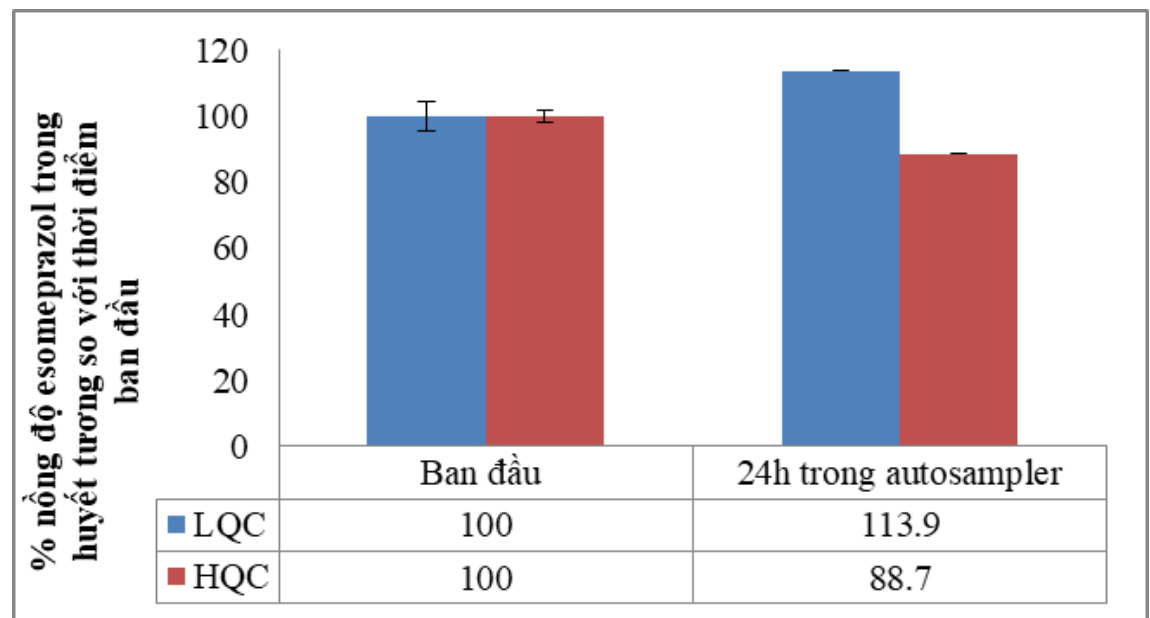

Hinh 6. Kết quả độ ổn định của mẫu sau chiết - bảo quản trong bộ tiêm mẫu tự động

Các giá trị RSD và độ lệch so với thời điểm ban đầu đều nhỏ hơn $15 \%$ cho thấy phương pháp đảm bảo được yêu cầu vê độ ổn định của dược chất trong huyết tương động vật thí nghiệm [9 - 11]. Tại nồng độ LQC, mức độ dao động về hàm lượng khá lớn, cho thấy sai số của phương pháp tại mức nồng độ này có dao động lớn; tuy nhiên sai số này vẫn nằm trong giới hạn cho phép $( \pm 15 \%)$.

\subsection{7. Üng dụng phương pháp phân tích esomeprazol trong huyết tương thỏ}

Áp dụng quy trình xử lý mẫu và phân tích bằng LC-MS/MS đã được khảo sát và thẩm định để phân tích định lượng esomeprazol trong huyết tương đối với chế phẩm chứa esomeprazol. Nhóm nghiên cứu đã áp dụng phương pháp trên để phân tích các mẫu huyết tương thu được 
tại các thời điểm xác định khi cho thỏ uống hai chế phẩm chứa esomeprazol. Từ đó xây dựng mô hình dược động học cho chế phẩm nghiên cứu bào chế và góp phân đánh giá khả năng hấp thu của chế phẩm nghiên cứu so với chế phẩm đối chiếu trên thị trường. Hình 7 thể hiện nông độ esomeprazol trong huyết tương thỏ theo thời gian.

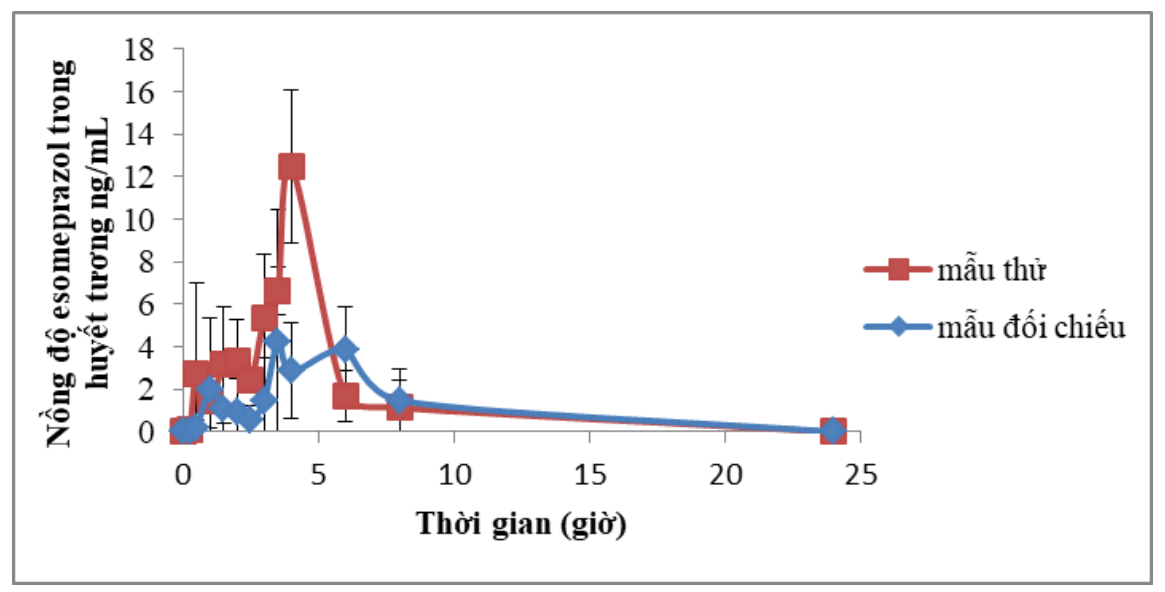

Hình 7. Nông độ esomeprazol trong huyết tương thỏ theo thời gian của chế phẩm nghiên cứu và chế phâm đối chiếu

Kết quả cho thấy thời gian đạt nồng độ cực đại $\left(\mathrm{T}_{\max }\right)$ của chế phẩm đối chiếu lớn hơn so với chế phẩm thử. Nông độ cực đại $\left(\mathrm{C}_{\max }\right)$ của chế phẩm micropellet bào chế cao gấp 2,24 lân so với $\mathrm{C}_{\max }$ của chế phẩm đối chiếu. Trong khi đó, diện tích dưới đường cong $\left(\mathrm{AUC}_{0-\infty}\right)$ của chế phẩm thử cao gấp 1,39 lần chế phẩm đối chiếu. Thời gian bán thải của chế phẩm đối chiếu là $1,367 \pm 0,888$ giờ, cao hơn chế phẩm thử là $0,962 \pm 0,535$ giờ. Qua các thông số dược động học cơ bản, chế phẩm thử cho tác dụng tốt hơn chế phẩm đối chiếu, công thức bào chế micropellet đang nghiên cứu đúng hướng và cần tiếp tục phát triển.

Các kết quả phân tích mẫu huyết tương tại các thời điểm đạt yêu cầu về độ đúng, độ chính xác và độ lệch theo quy định của phương pháp phân tích thuốc trong dịch sinh học (sai khác dưới $15 \%$ so với nông độ lý thuyết tương ứng) $[9,11]$. Như vậy, nghiên cứu đã trình bày về phương pháp định lượng esomeprazol trong huyết tương thỏ và được thẩm định đây đủ với quy trình xử lý mẫu theo phương pháp QuEChERS làm giảm ảnh hưởng của nền và tăng hiệu quả phương pháp, đặc biệt tiêu chí về độ ổn định đã được thẩm định là điểm khác so với những nghiên cứu trước đây, làm tăng độ tin cậy của phương pháp nghiên cứu trong việc ứng dụng đánh giá sinh khả dụng làm cơ sở để nghiên cứu bào chế phát triển thuốc mới chứa esomeprazol.

\section{KẾT LUẬN}

Sử dụng LC-MS/MS pha đảo kết hợp với xử lý mẫu bằng kỹ thuật QuEChERS, chúng tôi đã xây dựng được quy trình phân tích esomeprazol trong huyết tương thỏ có sử dụng chuẩn nội là pantoprazol với độ đặc hiệu đạt yêu câu ICH, U.S. FDA và AOAC, khoảng tuyến tính 0,1 - 20 $\mathrm{ng} / \mathrm{mL}$, độ đúng và độ chính xác với các mẫu nồng độ khảo sát đều đáp ứng theo yêu câu của ICH, độ thu hồi đạt yêu cầu AOAC, độ ổn định của esomeprazol trong huyết tương trong 03 chu kỳ đông - rã đông trong vòng 03 tháng và trong 24 giờ lưu giữ trong bộ tiêm mẫu đảm bảo yêu cầu với kết quả RSD và độ lệch so với thời điểm ban đâu nhỏ hơn $15 \%$. Phương pháp đã được ứng dụng để đánh giá sinh khả dụng của chế phẩm micropellet chứa esomeprazol so với chế phẩm đối chiếu. 


\section{LỜI CẢM ƠN}

Nhóm nghiên cứu xin gửi lời cảm ơn tới Dược sĩ Lê Thành Lâm công ty Cổ phần dược phẩm Mediplantex, công ty Colocon đã hỗ trợ nguyên vật liệu để thực hiện đề tài.

\section{TÀI LIỆU THAM KHẢO}

[1]. Astrazeneca, "Nexium • (esomeprazole magnesium) delayed - release capsules", 2006.

[2]. Medication guide nexium, 2018.

[3]. S. Missaghi, C. Young, K. Fegely, and A. R. Rajabi-Siahboomi, "Delayed release film coating applications on oral solid dosage forms of proton pump inhibitors: case studies," Drug development and industrial pharmacy, vol. 36, no. 2, pp. 180-189, 2010.

[4]. W. Talaat, "Bioanalytical method for the estimation of co-administered esomeprazole, leflunomide and ibuprofen in human plasma and in pharmaceutical dosage forms using micellar liquid chromatography," Biomedical Chromatography, vol. 31, no. 5, 2017.

[5]. R. H. B. Chunduri and G. S. Dannana, "Development and validation of a high throughput UPLC-MS/MS method for simultaneous quantification of esomeprazole, rabeprazole and levosulpiride in human plasma," Journal of Pharmaceutical Analysis, vol. 6, no. 3, pp. 190198, 2016.

[6]. E. F. Elkady, M. A. Fouad, and B. M. Jaadan, "LC-MS/MS bioassay of four proton pump inhibitors," Journal of Chromatography B: Analytical Technologies in the Biomedical and Life Sciences, vol. 1076, pp. 61-69, 2018.

[7]. M. Anastassiades, S. J. Lehotay, D. Stajnbaher, and F. J. Schenck, "Fast and easy multiresidue method employing acetonitrile extraction/partitioning and "dispersive solid-phase extraction" for the determination of pesticide residues in produce," Journal of AOAC International, vol. 86, no. 2, pp. 412-31, 2003.

[8]. United States Food and Drug Administration, Bioanalytical Method Validation Guidance for Industry, 2018.

[9]. ICH, ICH guideline M10 on bioanalytical method validation, 2019.

[10]. AOAC International, "Appendix F: Guidelines for Standard Method Performance," AOAC official methods of analysis, 2016.

[11]. B. Y. tế, Dược điên Việt Nam V, Phu luc 14 Hướng dẫn đánh giá sinh khả dụng và tương đương sinh học in vivo thuốc generic, Nhà xuất bản Y học, 2017.

[12]. United States Food and Drug Administration, Guidance for industry: estimating the maximum safe starting dose in initial clinical trials for therapeutics in adult healthy volunteers, 2015. 


\title{
Determination of esomeprazole in rabbit plasma by liquid chromatography tandem mass spectrometry (LC-MS/MS)
}

\author{
Le Minh Anh ${ }^{1}$, Nguyen Thach Tung ${ }^{1^{*}}$, Nguyen Nhu Nam ${ }^{1}$ \\ Bui Quang Dong ${ }^{2}$, Pham Thanh Dat ${ }^{1}$, Nguyen Thi Hong Ngoc ${ }^{2}$, Tran Cao Son ${ }^{2}$ \\ ${ }^{1}$ Hanoi University of Pharmacy, Hanoi, Vietnam \\ ${ }^{2}$ National Institute for Food Control, Hanoi, Vietnam
}

\section{Abstract}

A rapid extraction method was developed and validated for esomeprazole determination in rabbit plasma by liquid chromatography tandem mass spectrometry (LC-MS/MS). Esomeprazole in rabbit plasma was extracted and then cleaned up using QuEChERS technique. The analyte was separated using C18 column and quantified by MS/MS detector. Pantoprazole was used as the internal standard. The positive ESI source was used in this study together with the multi-reactive ion monitoring mode. The validity of the method has been confirmed in accordance with ICH Harmonized guidelines on bioanalytical method validation. The method showed good specificity, good stability, with the linearity varying from $0.1 \mathrm{ng} / \mathrm{mL}$ to $20 \mathrm{ng} / \mathrm{mL}$, the lower limit of quantification was $0.1 \mathrm{ng} / \mathrm{mL}$, the accuracy and precision were within $85 \%$ and $115 \%$ which achieved the ICH Harmonized guideline requirements, FDA guideline and AOAC International requirements. The method has been applied to quantify the concentration of esomeprazole in rabbit plasma, and then to compare the bioavailability of two preparations containing esomeprazole.

Keywords: Esomeprazole, Pantoprazole, LC-MS/MS, rabbit plasma, QuEChERS. 